Article

\title{
An Adaptive Single-Well Stochastic Resonance Algorithm Applied to Trace Analysis of Clenbuterol in Human Urine
}

\author{
Wei Wang ${ }^{1}$, Suyun Xiang ${ }^{2, *}$, Shaofei Xie ${ }^{3}$ and Bingren Xiang ${ }^{4}$ \\ 1 Department of Analytical Chemistry, China Pharmaceutical University, Nanjing 211198, China \\ 2 Jiangsu Key Laboratory for Supramolecular Medicinal Materials and Applications, \\ College of Life Science, Nanjing Normal University, Nanjing 210046, China \\ 3 Nanjing Changao Pharmaceutical Technology Limited, Nanjing 210022, China \\ 4 Key Laboratory of Drug Quality Control and Pharmacovigilance (Ministry of Education), \\ China Pharmaceutical University, Nanjing 210009, China \\ * Author to whom correspondence should be addressed; E-Mail: xiangsy@njnu.edu.cn; \\ Tel.: +86-25-85891591; Fax: +86-25-85891591.
}

Received: 13 December 2011; in revised form: 22 January 2012 / Accepted: 2 February 2012 / Published: 15 February 2012

\begin{abstract}
Based on the theory of stochastic resonance, an adaptive single-well stochastic resonance (ASSR) coupled with genetic algorithm was developed to enhance the signal-tonoise ratio of weak chromatographic signals. In conventional stochastic resonance algorithm, there are two or more parameters needed to be optimized and the proper parameters values were obtained by a universal searching within a given range. In the developed ASSR, the optimization of system parameter was simplified and automatic implemented. The ASSR was applied to the trace analysis of clenbuterol in human urine and it helped to significantly improve the limit of detection and limit of quantification of clenbuterol. Good linearity, precision and accuracy of the proposed method ensure that it could be an effective tool for trace analysis and the improvement of detective sensibility of current detectors.
\end{abstract}

Keywords: adaptive stochastic resonance algorithm; single-well; genetic algorithm; trace analysis; clenbuterol 


\section{Introduction}

Trace analysis is one of the routine tasks for analytical chemists in various fields of analytical chemistry. Highly sensitive detection techniques are helpful to reduce workload and enhance analysis accuracy. To pursue detection sensitivity, one approach is via hardware improvements, whereas an alternative approach is to enhance the signal-to-noise ratio (SNR) by chemometric methods. Various smoothing and filtering methods are widely used to improve the signal of interest by reducing the effect of noise [1-4]. However, they may result in loss (hopefully negligible) of useful information. Different from these methods, a stochastic resonance (SR) algorithm can amplify the weak signal significantly in a nonlinear system by making use of noise instead of filtering it [5-7]. Energy transfer from noise to useful signals will take place when signal and noise cooperate properly within the nonlinear system, i.e., the SR condition is reached, and accordingly, the SNR of useful signals will be enhanced.

For superior detection of useful signal submerged in heavy noise, the SR algorithm has received increasing interest from analytical chemists and has been successfully applied in pharmaceutical analysis [8,9], food analysis [10,11] and environmental analysis [12,13]. In the algorithm, a nonlinear system is one of the necessary factors for SR. The most frequently used nonlinear system in conventional SR is a bistable system described as a double-well potential. However, in the double-well potential stochastic resonance (DSR) system, two parameters named $a$ and $b$ are involved and need to be optimized for SR. In order to obtain an ideal output of the algorithm, additional parameters are often introduced into the system $[14,15]$, so there are two or more parameters and the optimizations are somewhat complicated. In 1993, Stocks replaced the bistable system with a monostable one with only one parameter [16]; and later, Zhang et al. developed a single-well potential stochastic resonance (SSR) with only one parameter to simply the algorithm [17].

The optimization of system parameters is essential for the application of the algorithm of SR. While in the previous studies, the optimal parameters were achieved by a universal one by one search within a given range, sometimes this was complicated and time consuming. A genetic algorithm (GA) is an adaptive heuristic search algorithm premised on the evolutionary ideas of natural selection and genetic [18-20]. It is a particular class of evolutionary algorithms that use techniques inspired by evolutionary biology such as inheritance, mutation, selection, and crossover. Being a population-based approach, GA is well suited to implement the automatic optimization of system parameters of the SR algorithm.

Clenbuterol is a selective adrenoceptor selective agonist and its main therapeutic use is in the treatment of pulmonary diseases for its longtime bronchodilator activity. Due to its similar physiological effects to sympathomimetic and anabolic steroids, the drug is popularly abused as a doping agent by athletes to increase performance, especially in bodybuilding, power-related and endurance sports. The abuse of clenbuterol hurts fair competition and athlete's health, so accordingly its use is prohibited both in and out of competition by the International Olympic Committee [21]. However, the use of masking agents, such as diuretics, makes the detection more difficult, so, a highly sensitive and reliable assay method is essential. Various methods have been used in the determination of clenbuterol in biological matrices. The reported methods include enzyme-linked immunosorbent assay (ELISA) [22-24], gas chromatography-mass spectrometry (GC/MS) [25,26], liquid 
chromatography-tandem mass spectrometry (LC/MS/MS) [27,28] and hapten microarrays [29]. Among these methods, some lack specificity for the possible cross-reactivity, some are timeconsuming due to complicated pretreatments and some need advanced detectors or special devices. The aim of the present work was to develop an adaptive single-well potential stochastic resonance algorithm (ASSR) coupled with GA for the trace analysis of clenbuterol in urine as a biological matrix. By applying the algorithm, the weak signal of clenbuterol is amplified significantly and those buried in noise could be determined accurately. The detection sensitivity is comparable or better than that of reported methods that require advanced detectors, and it may be expected that those advanced detectors would be more made more sensitive by the application of the proposed algorithm.

\section{Theory and Algorithm}

\subsection{Theory of Single-Well Potential Stochastic Resonance}

The nonlinear Langevin equation has often been used to describe the SR phenomenon. It has the following expression [30]:

$$
\frac{d x}{d t}=-U^{\prime}(x)+I(t)
$$

where the variable $x$ is the output of the nonlinear system, representing the position of signal. $I(t)=S(t)+N(t)$ denotes an input signal embedded in a noisy environment. $S(t)$ is the signal and $N(t)$ is the noise.

The single-well potential system in the algorithm of SSR developed by Zhang et al. [17] can be described by the following equation, where there's only one parameter $b$ defining the potential profile:

$$
U(x)=-a+\frac{1}{2} b x^{2}
$$

As shown in Figure 1, the single-well potential has no potential barrier. When SR takes place, the input signal will extract energy from the noise to move along the brim of the single-well potential. Thus, it can achieve a height that the single input signal cannot reach, and for this reason the strength of signals will increase and that of noise will decrease. Consequently, the output of a weak signal will give a better SNR than its input.

Figure 1. A typical single-well potential.

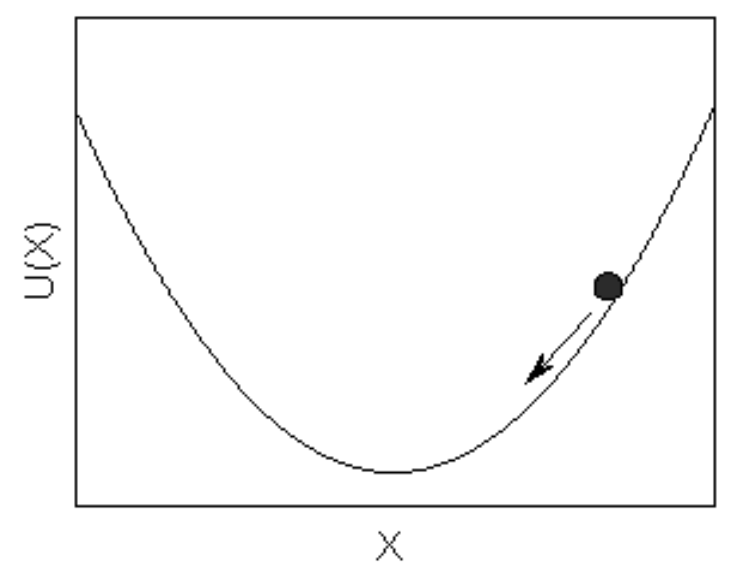


In the proposed SSR, the discrete stochastic differential equation [obtained by substitution of Eqution(3) into Eqution(1)] was solved by a fourth-order Runge-Kutta method [17]. The calculation procedure starts with normalization of the input signal $I(t)$ to the interval $[-1,1]$, and the normalized signal is then operated on by the algorithm to give the output signal. The final results can be obtained by inverse normalization of the output signals.

\subsection{Genetic Algorithm}

When the algorithm of SSR applied to quantitative determination, the optimization of $b$ is essential for this parameter will affect the profile of the potential well and the final output results. In this work, the parameter $b$ was optimized to give out a maximal SNR of the output signal. Here, the objective function of the SSR model can be defined as follows:

$$
\begin{cases}\min & f(b)=-\frac{S N R(b)}{S N R_{\max }} \\ \text { s.t. } & 0<b \leq 1\end{cases}
$$

where $S N R(b)$ is the SNR of system output signal and $S N R_{\max }$ is the maximal SNR with a different parameter $b$.

GA is a commonly used approach to solving optimization problems. Here, vector evaluated GA (or VEGA) proposed by Schaffer [31] was used to implement the automatic optimization of the parameter $b$. In VEGA, a population $p_{t}$ is randomly divided into $\mathrm{K}$ equal sized subpopulations: $p_{1}, p_{2}, \ldots, p_{K}$. Then, each solution in subpopulation $p_{i}$ is assigned a fitness value based on objective function $z_{i}$. Solutions are selected from these subpopulations using proportional selection for crossover and mutation. Crossover and mutation are performed on the new population in the same way.

In this paper, the parameter $b$ of SSR was optimized by VEGA. The procedures of optimization are as follows:

$N_{s}=$ subpopulation size $\left(N_{s}=N / K\right)$.

Step 1: Start with a random initial population $P_{0}$. Set $t=0$.

Step 2: If the stopping criterion is satisfied, return $P_{t}$.

Step 3: Randomly sort population $P_{t}$.

Step 4: For each objective $k, k=1, \ldots, \mathrm{K}$, perform the following steps:

Step 4.1: For $i=1+(k-1) N_{s}, \ldots, k N_{s}$, assign fitness value $f\left(x_{i}\right)=z_{k}\left(x_{i}\right)$ to the $i$ th solution in the sorted population.

Step 4.2: Based on the fitness values assigned in Step 4.1, select $N_{s}$ solutions between the $\left(1+(k-1) N_{s}\right)$ th and $\left(k N_{s}\right)$ th solutions of the sorted population to create subpopulation $P_{k}$.

Step 5: Combine all subpopulations $P_{1}, \ldots, P_{k}$ and apply crossover and mutation on the combined population to create $P_{t+1}$ of size $N$. Set $t=t+1$, go to Step 2 .

The parameters used in implementing GA are as follows:

Number of individuals: 200;

Maximum number of generations: 100;

Precision of variables: 20;

Generation gap: 0.7; 
All the calculations were performed using programs written by the authors in the Matlab 7.0 environment (Mathworks, Natick, MA, USA), running on a PC with an Intel(R) Core Duo CPU 1.83 GHz and $1 \mathrm{G}$ RAM.

\section{Results and Discussion}

\subsection{Optimization of System Parameter}

When the input signal is fixed, the nonlinear system parameters will directly influence the quality of final output signal and consequently influence the results of quantitative determination. A raw chromatogram of clenbuterol at a concentration of $0.2 \mathrm{ng} / \mathrm{mL}$ with an original SNR of 6.1 was used for the optimization. As a result of optimization, a maximal SNR of 22.6 was obtained when $b=0.0574$.

\subsection{Quantitative Analysis of Clenbuterol in Urine}

Although the raw intensities of clenbuterol vary with the spiked concentrations in target samples, the same parameter will be used throughout to keep the quantitative relationship of the output signals. A set of spiked urine samples with clenbuterol at different concentration levels were prepared and assayed, and then the chromatograms were processed by ASSR with the optimized parameter ( $b=0.0574)$ developed above.

The specificity of the assay was studied by using blank urine samples. No endogenous interferences were observed both before and after applying the algorithm. Signal-to-noise ratio of 3 and 10 are usually considered as limit of detection (LOD) and limit of quantification (LOQ), respectively. The LOD and LOQ of LC-MS analysis of clenbuterol can be estimated at $0.1 \mathrm{ng} / \mathrm{mL}$ and $0.3 \mathrm{ng} / \mathrm{mL}$, respectively. With the application of ASSR, the LOD and LOQ were respectively improved to $0.025 \mathrm{ng} / \mathrm{mL}$ and $0.05 \mathrm{ng} / \mathrm{mL}$. The raw chromatogram of LOD is shown in Figure 2(a) and (b) wherein the clenbuterol peak is buried in noise. It is obvious that the weak signal is hard to detect. After being processed by ASSR, the output signal of clenbuterol showed a significant SNR improvement [shown in Figure 2c].

Figure 2. Chromatogram of urine spiked with clenbuterol at $0.025 \mathrm{ng} / \mathrm{mL}$. (a) Full length of the chromatogram. (b) Especially enlarged chromatogram of clenbuterol. (c) The peak of clenbuterol obtained by ASSR with $b=0.0574$.

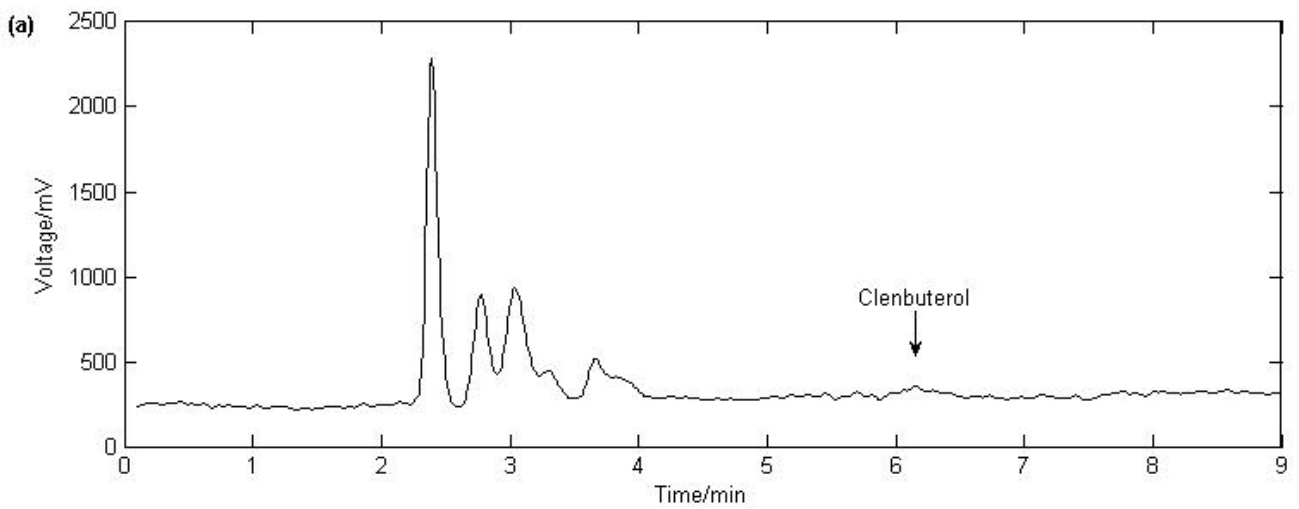


Figure 2. Cont.
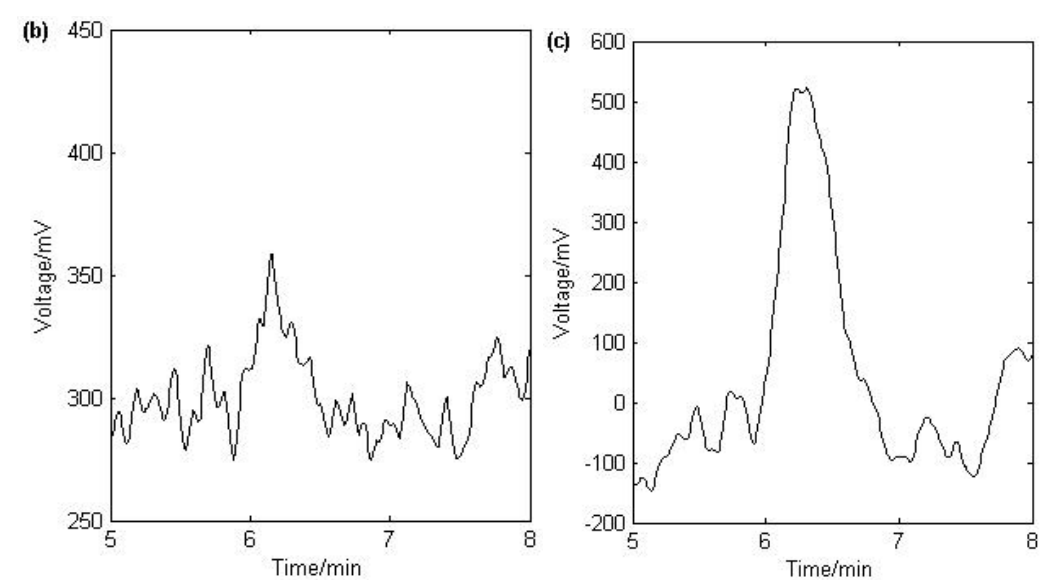

The calibration curve was prepared and assayed with the urine samples spiked with clenbuterol at concentrations ranging from $0.2 \mathrm{ng} / \mathrm{mL}$ to $20.0 \mathrm{ng} / \mathrm{mL}$. Then the raw chromatograms of clenbuterol were processed with the optimized ASSR. A comparison is made between the raw chromatograms and those obtained by ASSR in Figure 3.

Figure 3. Chromatograms of clebuterol in urine samples of calibration curve. (a) The raw chromatograms of clenbuterol. (b) The chromatograms of clenbuterol obtained by ASSR with $\boldsymbol{b}=0.0574$. From left to right, the concentrations of clenbuterol are 0.2, 0.5, 1.0, 2.0, $5.0,10.0$ and $20.0 \mathrm{ng} / \mathrm{mL}$, respectively.

(a)

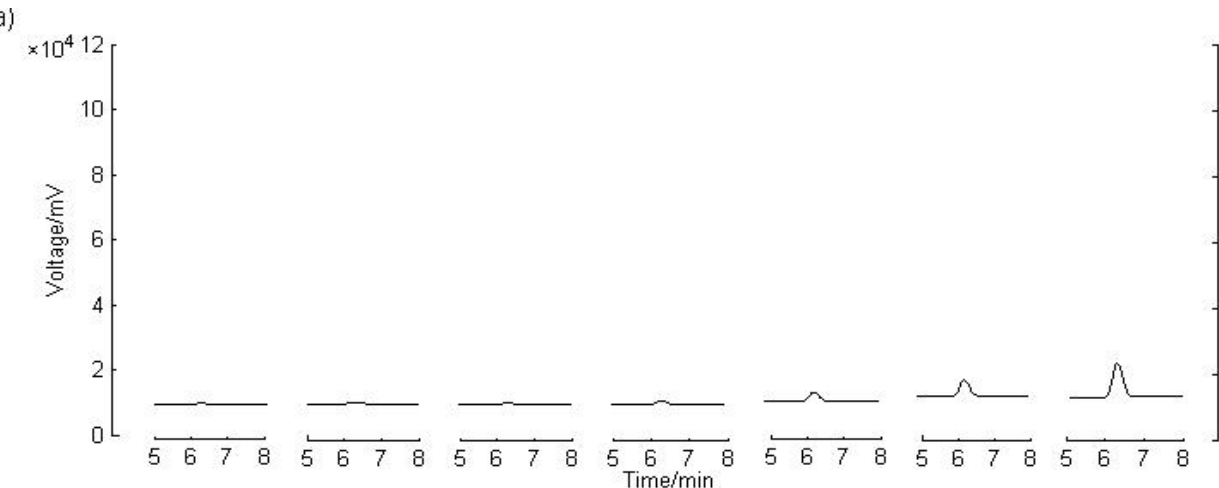

(b)

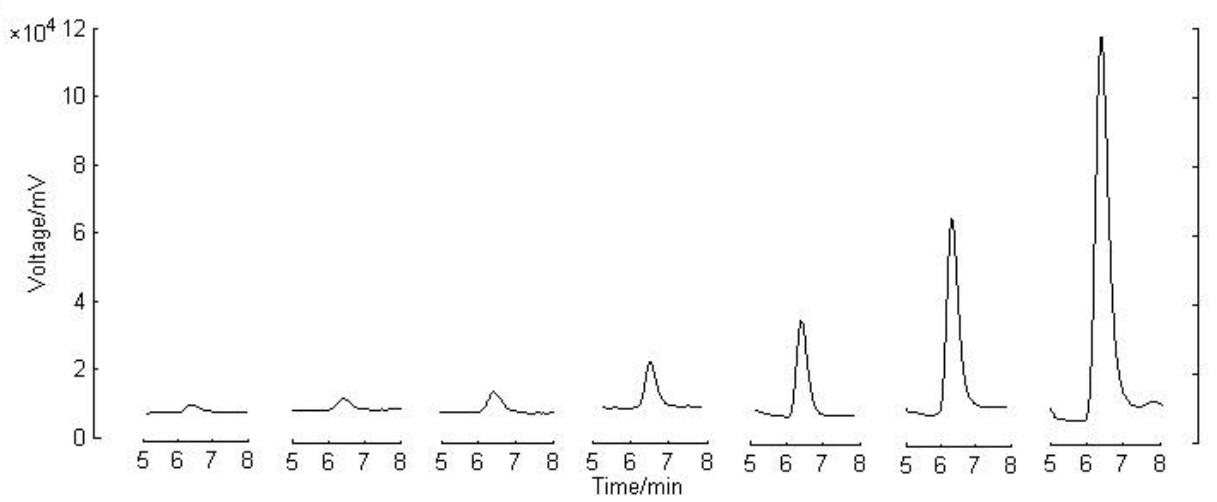

The calculated curve illustrated in Table 1 displays a good linear relationship between the peak area ratio $(f)$ of clenbuterol to IS and clenbuterol concentration. The results show that all the original signals of target analyte are significantly enhanced and maintain a good linear relationship as well. 
Table 1. Calibration curve of clenbuterol by ASSR.

\begin{tabular}{lccccccc}
\hline Conc. $(\mathbf{n g} / \mathbf{m L})$ & $\mathbf{0 . 2}$ & $\mathbf{0 . 5}$ & $\mathbf{1}$ & $\mathbf{2}$ & $\mathbf{5}$ & $\mathbf{1 0}$ & $\mathbf{2 0}$ \\
\hline Ratio $(f)$ & 0.243 & 0.465 & 0.917 & 1.689 & 3.311 & 6.918 & 14.716 \\
Calibration Curve & & & $f=0.722 \mathrm{C}+0.0453 \mathrm{r}=0.999$ & & \\
\hline
\end{tabular}

The investigation of precision and accuracy was carried out with spiked preparations at concentration of $0.5,2.0$ and $10.0 \mathrm{ng} / \mathrm{mL}$. Intra-day precision was determined by repeated analysis of urine samples within one day $(n=6)$, and inter-day precision was determined by repeated analysis of urine samples on three consecutive days ( $n=6$ series per day). The method accuracy was testing from recovery analysis which was defined as the ratio of the measured concentration to the added concentration. The intra- and inter-day assays are summarized in Table 2 and the results indicate that the accuracy and precision of the assay are acceptable.

Table 2. Acurracy and precision of clenbuterol by ASSR.

\begin{tabular}{|c|c|c|c|c|c|c|}
\hline \multirow[b]{2}{*}{$\begin{array}{c}\text { Added } \\
\text { Conc. } \\
(\mathrm{ng} / \mathrm{mL})\end{array}$} & \multicolumn{3}{|c|}{ Intra-day assay } & \multicolumn{3}{|c|}{ Inter-day assay } \\
\hline & $\begin{array}{c}\text { Measured Conc. } \\
(\text { mean } \pm \text { S.D. }) \\
(n g / m L)\end{array}$ & $\begin{array}{l}\text { Precision } \\
\text { R.S.D (\%) }\end{array}$ & $\begin{array}{c}\text { Accuracy } \\
(\%)\end{array}$ & $\begin{array}{l}\text { Measured Conc. } \\
(\text { mean } \pm \text { S.D. }) \\
(\mathbf{n g} / \mathbf{m L})\end{array}$ & $\begin{array}{c}\text { Precision } \\
\text { R.S.D } \\
(\%)\end{array}$ & $\begin{array}{c}\text { Accuracy } \\
\text { (\%) }\end{array}$ \\
\hline 0.5 & $0.55 \pm 0.04$ & 7.6 & 109.5 & $0.56 \pm 0.05$ & 8.7 & 111.9 \\
\hline 2.0 & $2.07 \pm 0.12$ & 5.8 & 103.5 & $1.87 \pm 0.14$ & 7.3 & 93.4 \\
\hline 10.0 & $9.60 \pm 0.53$ & 5.5 & 96.0 & $9.87 \pm 0.55$ & 5.6 & 98.7 \\
\hline
\end{tabular}

\section{Experimental}

\subsection{Materials and Reagents}

Standards of clenbuterol and terazosin (used as internal standard) were obtained from the National Institute for the Control of Pharmaceutical and Biological Products (Beijing, China). HPLC grade methanol and acetonitrile were purchased from Merck (Germany). Acetic acid and ammonium hydroxide, both analytical grade, were obtained from Nanjing Chemical Reagent Co., Ltd. (Nanjing, China). Double distilled water was used throughout the study. SCX SPE (250 mg) and C18 SPE (250 mg) disposable cartridges (Hanbon Sci. \& Tech., Jiangsu, China) were used for sample pretreatment and purification.

\subsection{Standard Solution}

Standard stock solutions at $1.0 \mathrm{mg} / \mathrm{mL}$ of clenbuterol and terazosin were prepared in methanol and stored at $4{ }^{\circ} \mathrm{C}$. Working solutions of clenbuterol $(10,100$ and 1,000 ng/mL) and terazosin $(1,000 \mathrm{ng} / \mathrm{mL})$ were prepared by dilution with methanol.

\subsection{Sample Preparation}

$\mathrm{C}_{18}$ SPE cartridge and SCX SPE cartridge fixed in downward mode were conditioned successively with methanol, water and $30 \mathrm{mmol} / \mathrm{L}$ hydrochloric acid $(5.0 \mathrm{~mL}$ each). Human urine $(5 \mathrm{~mL})$ spiked 
with clenbuterol and internal standard (terazosin) was adjusted to about $\mathrm{pH} 5.3$ with acetic acid (200 $\mu \mathrm{L}, 1 \%, \mathrm{v} / \mathrm{v}$ ), vortex mixed for $5 \mathrm{~s}$ and loaded onto the $\mathrm{C}_{18}$ cartridge. Then cartridge was washed with water $(5 \mathrm{~mL})$ and methanol $(5 \mathrm{~mL})$, then the $\mathrm{C}_{18}$ cartridge was removed, and the SCX cartridge was eluted with methanol $\left(5 \mathrm{~mL}, 4 \%\right.$ ammonia, v/v). Eluates were evaporated with nitrogen at $50{ }^{\circ} \mathrm{C}$, and the residues were reconstituted with acetonitrile $(200 \mu \mathrm{L})$.

\subsection{LC/MS Analysis}

The analysis was performed on an HP1100 LC/MSD system (Hewlett-Packard, USA), with the liquid chromatography part consisting of an automatic sampler and a binary pump with an online degasser, and the mass spectrometry unit consisting of a single quadrupole mass spectrometer equipped with an electrospray ionization interface (ESI). The separation was carried on a Diamonsil $\mathrm{C}_{18}$ column $(250 \times 4.6 \mathrm{~mm}$, i.d.; $5 \mu \mathrm{m}$, Dikma, China $)$ with temperature set at $25^{\circ} \mathrm{C}$. The mobile phase consisted of $0.02 \mathrm{M}$ ammonium formate buffer $(\mathrm{pH} 3.5)$-acetonitrile $(72: 28, \mathrm{v} / \mathrm{v})$ at a flow rate of $1.0 \mathrm{~mL} / \mathrm{min}$. Mass spectrometric detection was operated in the positive mode with the fragmentor voltage set at $70 \mathrm{~V}$ and capillary voltage at $4 \mathrm{kV}$, drying gas $\left(\mathrm{N}_{2}\right)$ flow of $10.5 \mathrm{~L} / \mathrm{min}$ and temperature of $350{ }^{\circ} \mathrm{C}$, nebulizer pressure of $45 \mathrm{psi}$. The $[\mathrm{M}+\mathrm{H}]^{+}, \mathrm{m} / z 277$ for clenbuterol and $[\mathrm{M}+\mathrm{H}]^{+}, \mathrm{m} / z 388$ for terazosin were selected as detection ions, respectively.

\section{Conclusions}

An adaptive single-well stochastic resonance algorithm was developed for trace analysis. In the proposed algorithm, there is only one system parameter and this parameter was automatic optimized by a genetic algorithm. The simplified optimization progress makes the algorithm easier to manipulate in practice. By applying the algorithm, weak signals of clenbuterol were amplified significantly, the LOD and LOQ were improved from $0.1 \mathrm{ng} / \mathrm{mL}$ to $0.025 \mathrm{ng} / \mathrm{mL}$ and from $0.3 \mathrm{ng} / \mathrm{mL}$ to $0.05 \mathrm{ng} / \mathrm{mL}$, respectively. It could be expected those advanced detectors coupled with the proposed algorithm would be more effective for trace analysis.

\section{Acknowledgements}

This work was supported by the Natural Science Foundation of the Jiangsu Higher Education Institutions of China (Grant No. 11KJD350001) and the Research Fund for the Doctoral Program of Higher Education of China (Grant No. 20113207120007).

\section{References and Notes}

1. Permann, D.N.S.; Teitelbaum, H. Wavelet fast Fourier transform (WFFT) analysis of a millivolt signal for a transient oscillating chemical reaction. J. Phys. Chem. 1993, 97, 12670-12673.

2. Muddiman, D.C.; Huang, B.M.; Anderson, G.A.; Rockwood, A.; Hofstadler S.A.; Weir-Lipton M.S.; Proctor, A.; Qinyuan, W.; Smith, R.D. Application of sequential paired covariance to liquid chromatography-mass spectrometry data Enhancements in both the signal-to-noise ratio and the resolution of analyte peaks in the chromatogram. J. Chromatogr. A 1997, 771, 1-7. 
3. Vivó-Truyols, G.; Schoenmakers, P.J. Automatic selection of optimal Savitzky-Golay smoothing. Anal. Chem. 2006, 78, 4598-4608.

4. Wen, X.; Zhang, H.; Wang, F. A Wavelet Neural Network for SAR Image Segmentation. Sensors 2009, 9, 7509-7515.

5. Pan, Z.; Guo, W.; Wu, X.; Cai, W.; Shao, S. A new stochastic resonance algorithm to improve the detection limits for trace analysis. Chemom. Intell. Lab. Syst. 2003, 66, 41-49.

6. Goris, R.L.T.; Wagemans, J.; Wichmann, F.A. Modelling contrast discrimination data suggest both the pedestal effect and stochastic resonance to be caused by the same mechanism. J. Vis. 2008, 8, 17.1-17.21.

7. Rallabandi, V.P.; Roy, P.K. Magnetic resonance image enhancement using stochastic resonance in Fourier domain. Magn. Reson. Imaging 2010, 28, 1361-1373.

8. Zhang, W.; Xiang, B.; Wu, Y.; Shang, E. Stochastic resonance is applied to quantitative analysis for weak chromatographic signal of glyburide in plasma. Anal. Chim. Acta 2005, 550, 77-81.

9. Xiang, S.; Wang, W.; Xiang, B.; Deng, H.; Xie, S. Periodic modulation-based stochastic resonance algorithm applied to quantitative analysis for weak liquid chromatography-mass spectrometry signal of granisetron in plasma. Int. J. Mass Spectrom. 2007, 262, 174-179.

10. Ye, Y.; Xiang, B.; Zhang, W.; Shang, E. Stochastic resonance is applied to quantitative analysis for weak chromatographic signal of Sudan I. Phys. Lett. A 2006, 359, 620-623.

11. Zhan,Y.; Xiang, B.; Zhang, W.; Xie, S.; Deng, H.; Xiang, S. Single-well potential stochastic resonance applied to quantitative analysis for weak chromatographic signals of sudan IV. Anal. Lett. 2008, 40, 2415-2424.

12. Xie, S.; Deng, H.; Xiang, B.; Xiang, S. Detection of trace triclocarban in water sample using solid-phase extraction-liquid chromatography with stochastic resonance slgorithm. Environ. Sci. Technol. 2008, 42, 2988-2991.

13. Xiang, S.; Wang, W.; Xia, J.; Xiang, B.; Ouyang, P. Stochastic resonance algorithm applied to quantitative analysis for weak chromatographic signals of alkyl halides and alkyl benzenes in water samples. J. Chromatogr. Sci. 2009, 47, 700-704.

14. Pan, Z.X.; Wu, X.J.; Guo, W.M.; Cai, W.S.; Shao, X.G. Studies on the detection of weak chemical signal by using a stochastic resonance algorithm based on periodic modulation. Chem. Res. Chinese U. 2003, 24, 605-608.

15. Deng, H.; Shang, E.; Xiang, B.; Xie, S.; Tang, Y.; Duan, J.; Zhan, Y.; Chi, Y.; Tan, D. Application of the stochastic resonance algorithm to the simultaneous quantitative determination of multiple weak peaks of ultra-performance liquid chromatography coupled to time-of-flight mass spectrometry. Rapid Commun. Mass Spectrom. 2011, 25, 563-571.

16. Stocks, N.G.; Stein, N.D.; McClintock, P.V.E. Stochastic resonance in monostable systems. J. Phys. A: Math. Gen. 1993, 26, L385-L390.

17. Zhang, W.; Xiang, B. A new single-well potential stochastic resonance algorithm to detect the weak signal. Talanta 2006, 70, 267-271.

18. Liu, F.; Wang, J. Genetic algorithms and its application to spectral analysis. Guang Pu Xue Yu Guang Pu Fen Xi 2001, 21, 331-335.

19. Dugan, N.; Erkoç, Ş. Genetic algorithms in application to the geometry optimization of nanoparticles. Algorithms 2009, 2, 410-428. 
20. Asadollahi, T.; Dadfarnia, S.; Shabani, A.M.H.; Ghasemi, J.B.; Sarkhosh, M. QSAR Models for CXCR2 Receptor Antagonists Based on the Genetic Algorithm for Data Preprocessing Prior to Application of the PLS Linear Regression Method and Design of the New Compounds Using In Silico Virtual Screening. Molecules 2011, 16, 1928-1955.

21. World Anti-Doping Agency. The World Anti-Doping Code. The 2011 prohibited list international standard, 1 January 2011. Available online: http://www.wada-ama.org/Documents/World_AntiDoping_Program/WADP-Prohibited-list/To_be_effective/WADA_Prohibited_List_2011_EN.pdf (accessed on 22 April 2011).

22. Lai, W.H.; Fung, D.Y.; Xu, Y.; Xiong, Y.H. Screening procedures for clenbuterol residue determination in raw swine livers using lateral-flow assay and enzyme-linked immunosorbent assay. J. Food Prot. 2008, 71, 865-869.

23. Liu, N.; Su, P.; Gao, Z.; Zhu, M.; Yang, Z.; Pan, X.; Fang, Y.; Chao, F. Simultaneous detection for three kinds of veterinary drugs: Chloramphenicol,clenbuterol and 17-beta-estradiol by high-throughput suspension array technology. Anal. Chim. Acta 2009, 632,128-134.

24. Pleadin, J.; Vulić, A.; Mitak, M.; Perši, N.; Milić, D. Determination of clenbuterol residues in retinal tissue of food-producing pigs. J. Anal. Toxicol. 2011, 35, 28-31.

25. He, L.; Su, Y.; Zeng, Z.; Liu, Y.; Huang, X. Determination of ractopamine and clenbuterol in feeds by gas chromatography-mass spectrometry. Anim. Feed Sci. Tech. 2007, 132, 316-323.

26. Gallo, P.; Brambilla, G.; Neri, B.; Fiori, M.; Testa, C.; Serpe, L. Purification of clenbuterol-like $\beta 2$-agonist drugs of new generation from bovine urine and hair by $\alpha 1$-acid glycoprotein affinity chromatography and determination by gas chromatography-mass spectrometry. Anal. Chim. Acta 2007, 587, 67-74.

27. Li, C.; Wu, Y.L.; Yang, T.; Zhang, Y.; Huang-Fu, W.G. Simultaneous determination of clenbuterol, salbutamol and ractopamine in milk by reversed-phase liquid chromatography tandem mass spectrometry with isotope dilution. J. Chromatogr. A 2010, 1217, 7873-7877.

28. Chen, X.; Wu, Y.; Yang, T. Simultaneous determination of clenbuterol, chloramphenicol and diethylstilbestrol in bovine milk by isotope dilution ultraperformance liquid chromatographytandem mass spectrometry. J. Chromatogr. B 2011, 879, 799-803.

29. Zuo, P.; Zhang, Y.; Liu, J.; Ye, B.C. Determination of beta-adrenergic agonists by hapten microarray. Talanta 2010, 82, 61-66.

30. Gammaitoni, L.; Hanggi, P.; Jung, P.; Marchesoni, F. Stochastic resonance. Rev. Modern Phys. 1998, 70, 223-287.

31. Schaffer, J.D. Multiple objective optimization with vector evaluated genetic algorithms. In Proceedings of the International Conference on Genetic Algorithms and their Applications, Pittsburgh, PA, USA, 24-26 July 1985.

Sample Availability: Contact the authors.

(C) 2012 by the authors; licensee MDPI, Basel, Switzerland. This article is an open access article distributed under the terms and conditions of the Creative Commons Attribution license (http://creativecommons.org/licenses/by/3.0/). 